\title{
Thermodynamic Analysis of Ligand-Induced Changes in Protein Thermal Unfolding Applied to High-Throughput Determination of Ligand Affinities with Extrinsic Fluorescent Dyes ${ }^{\dagger}$
}

\author{
Curtis J. Layton and Homme W. Hellinga* \\ Department of Biochemistry, Duke University Medical Center, Durham, North Carolina 27710, United States
}

Received September 1, 2010; Revised Manuscript Received October 29, 2010

\begin{abstract}
The quantification of protein-ligand interactions is essential for systems biology, drug discovery, and bioengineering. Ligand-induced changes in protein thermal stability provide a general, quantifiable signature of binding and may be monitored with dyes such as Sypro Orange (SO), which increase their fluorescence emission intensities upon interaction with the unfolded protein. This method is an experimentally straightforward, economical, and high-throughput approach for observing thermal melts using commonly available real-time polymerase chain reaction instrumentation. However, quantitative analysis requires careful consideration of the dye-mediated reporting mechanism and the underlying thermodynamic model. We determine affinity constants by analysis of ligand-mediated shifts in melting-temperature midpoint values. Ligand affinity is determined in a ligand titration series from shifts in free energies of stability at a common reference temperature. Thermodynamic parameters are obtained by fitting the inverse first derivative of the experimental signal reporting on thermal denaturation with equations that incorporate linear or nonlinear baseline models. We apply these methods to fit protein melts monitored with SO that exhibit prominent nonlinear post-transition baselines. SO can perturb the equilibria on which it is reporting. We analyze cases in which the ligand binds to both the native and denatured state or to the native state only and cases in which protein:ligand stoichiometry needs to treated explicitly.
\end{abstract}

The interaction of proteins with ligands is a fundamental aspect of biomolecular function. The quantification of such interactions is essential for systems biology, drug discovery, and bioengineering. Traditional, generalizable methods for measuring protein-ligand affinity such as equilibrium dialysis or isothermal titration calorimetry require large amounts of material or radiolabeled ligands. Ligand-induced changes in protein stability also provide a general, quantifiable signature of protein-ligand interactions $(1-6)$, and hence of biological function (7). The measurement of protein stability typically also has required relatively large amounts of protein and low-throughput instrumentation and, consequently, has not been widely used as a tool to assess function. However, recently a number of techniques that allow protein stabilities to be determined with small amounts of material in a high-throughput manner have been developed $(8-12)$. One such method is based on extrinsic fluorescent dyes that monitor protein (un)folding $(2,3,13)$. This technique uses the relatively inexpensive fluorescent dye Sypro Orange $(\mathrm{SO})^{1}(14)$ in combination with readily available real-time polymerase chain reaction (RT-PCR) instrumentation $(3,13,15)$ and is being adopted as a straightforward, economical, high-throughput screening tool for ligand discovery in the pharmaceutical industry $(4,15)$, structural genomics efforts $(16,17)$, and high-throughput

\footnotetext{
${ }^{\dagger}$ This work was supported by a National Institutes of Health Director's Pioneer Award (5DPI OD000122).

*To whom correspondence should be addressed. Phone: (919) 6815885. Fax: (919) 684-8885. E-mail: hwh@ biochem.duke.edu.

${ }^{1}$ Abbreviations: SO, Sypro Orange; SN, staphylococcal nuclease A; MBP, Escherichia coli maltose-binding protein; RBP, E. coli ribosebinding protein; pdTp, thymidine $3^{\prime}, 5^{\prime}$-diphosphate; DMSO, dimethyl sulfoxide; $\mathrm{DMF}$, dimethylformamide; $\mathrm{CD}$, circular dichroism.
}

protein engineering (18). Here we present insights into the properties of the reporter dye relevant to the mechanism of reporting on protein denaturation and provide an approach for fitting the experimental data using a thermodynamic framework for quantifying free energies of stability and binding. This effort builds on previous work $(2-4,13)$ and addresses a number of experimental regimes.

Although SO has been used successfully to collect thermal denaturation data and characterize binding affinity constants by thermal shift (2-4), its properties have not been characterized extensively. Here we show that SO exhibits two unanticipated properties. First, in protein-free aqueous solutions, SO fluorescence emission intensity peaks at a critical dye concentration, which we ascribe to a self-aggregation phenomenon. Second, SO can significantly perturb the equilibria on which it is reporting in a concentration-dependent manner.

For the analysis of experimental data, the fluorescence intensities monitoring protein (un)folding are transformed into the inverse first-derivative form, such that the (un)folding transition manifests itself as a prominent trough, the minimum and width of which are directly related to the transition midpoint temperature and enthalpy of (un)folding (Figures 2 and 9). To obtain thermodynamic parameters, we fit these observations to an equation describing the temperature dependence of the experimental fluorescence signal that directly incorporates functional forms (linear or nonlinear) of the temperature dependence of the native (pretransition) and unfolded (post-transition) states. We present straightforward experimental procedures for estimating heat capacities, allowing a complete thermodynamic analysis from multiple observations. To apply these methods to realistic 
experimental cases, we have considered three different scenarios: (1) the ligand binding to both the native and denatured state, (2) the ligand binding to the native state only, and (3) ligand and protein concentrations requiring that stoichiometry be taken into account.

\section{MATERIALS AND METHODS}

Protein Purifications. Staphylococcal nuclease A (SN), Escherichia coli maltose-binding protein (MBP), and E. coli ribose-binding protein (RBP) were expressed (for sequences, see the Supporting Information) and purified from an E. coli culture in autoinduction medium essentially as described by Isom et al. (manuscript in press in Proteins: Structure, Function, and Bioinformatics). Protein concentrations were estimated from absorbance at $280 \mathrm{~nm}$ using calculated extinction coefficients (19) (for SN, $\varepsilon_{280}=15930 \mathrm{M}^{-1} \mathrm{~cm}^{-1}$; for MBP, $\varepsilon_{280}=70820 \mathrm{M}^{-1} \mathrm{~cm}^{-1}$; for RBP, $\varepsilon_{280}=4470 \mathrm{M}^{-1} \mathrm{~cm}^{-1}$ ).

Monitoring of Protein Unfolding and Ligand Binding with Sypro Orange. SN was used at a final concentration of $6 \mu \mathrm{M}, \mathrm{MBP}$ at $2 \mu \mathrm{M}$, and $\mathrm{RBP}$ at $6 \mu \mathrm{M}$. Experiments were conducted in $25 \mathrm{mM}$ potassium phosphate (SN, pH 7; MBP and RBP, pH 7.5) and $\mathrm{KCl}(\mathrm{SN}, 100 \mathrm{mM}$; MBP and RBP, $140 \mathrm{mM}$ ); mixtures for SN experiments additionally contained $500 \mu \mathrm{M}$ EDTA to chelate any residual $\mathrm{Ca}^{2+}$. Sypro Orange (Invitrogen catalog no. S6651) was used at a final concentration of $20 \times$, unless otherwise indicated. To take measurements, each sample was subdivided into either two or four $20 \mu \mathrm{L}$ replicates on a 384-well plate and fluorescence measurements were taken on a Roche LightCycler 480 II RT-PCR machine with filters to excite at $465 \mathrm{~nm}$ and measure emission at $580 \mathrm{~nm}$ as the temperature was continuously increased (ramp rates for determination of ligand affinities, $9.0^{\circ} \mathrm{C} / \mathrm{min}$ for the $\mathrm{SN}-$ pdTp pair and $0.5^{\circ} \mathrm{C} / \mathrm{min}$ for the $\mathrm{MBP}-$ maltose pair). Inverse derivative curves were exported directly from the machine and fit with custom software. Replicate $T_{\mathrm{m}}$ and $\Delta H_{\mathrm{u}}$ values were averaged. The concentration of pdTp (Toronto Research Chemicals catalog no. T410000) was determined by absorbance at $267 \mathrm{~nm}$ using an extinction coefficient of $9700 \mathrm{M}^{-1} \mathrm{~cm}^{-1}(20)$.

\section{RESULTS}

Thermodynamic Analysis of Thermal Shift Data. In the presence of any ligand that preferentially binds the native state, a binding-induced increase in protein stability can be observed as an increase in the melting-temperature midpoint $\left(T_{\mathrm{m}}\right)$. The values of $K_{\mathrm{d}}$ and $\Delta G_{\mathrm{b}}$ are obtained from thermal denaturations performed at varying ligand concentrations, which are analyzed with a thermodynamic model of the linkage between binding and stability. Quantitative analysis of two-state thermal denaturation with an equilibrium model is based on an experimentally observed signal, $\sigma(T)$, which reports on the equilibrium fraction of (un)folded protein. The temperature dependence of two-state (un)folding is described by the transition between the pre- and post-transition baselines, and the total signal observed during the thermal denaturation can be written

$$
\sigma(T)=\beta_{\mathrm{N}}(T) f(T)+\beta_{\mathrm{D}}(T)[1-f(T)]
$$

where $f(T)$ is the fraction folded at temperature $T$ and $\beta_{\mathrm{N}}(T)$ and $\beta_{\mathrm{D}}(T)$ are functions that describe the baseline signal from the native and denatured protein, respectively. The temperature at which $f(T)=0.5$ is defined as the $T_{\mathrm{m}}$ of the denaturation. Typically, baselines are linear, as is the case for folding transitions

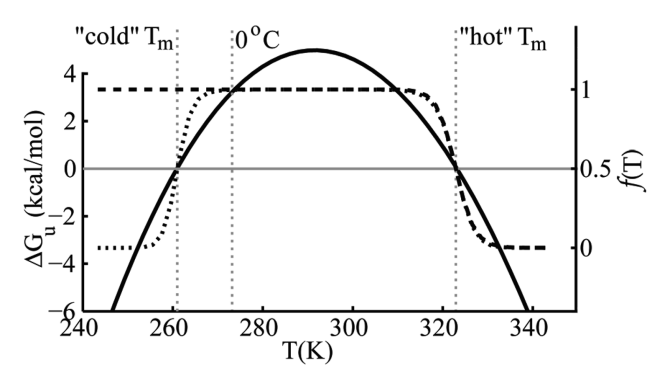

FIGURE 1: Comparison of equations describing the fraction of folded protein. The left ordinate shows the Gibbs-Helmholtz curve (eq 5) showing protein stability as a function of temperature $(-)$. The right ordinate shows the corresponding fraction folded according to eq $7(\cdots)$ and eq $9(--)$.

monitored by circular dichroism (CD). However, in the case of transitions monitored by SO, we find that some baselines are better represented by other, nonlinear functional forms (see below).

To analyze the temperature dependence of the observed folded fraction, we use the temperature-dependent equilibrium constant of two-state folding $\left(K_{\mathrm{u}}=[\mathrm{D}] /[\mathrm{N}]\right)$ :

$$
f(T)=\frac{1}{1+K_{\mathrm{u}}(T)}
$$

which is related to the free energy of unfolding by

$$
K_{\mathrm{u}}(T)=\mathrm{e}^{-\left[\Delta G_{\mathrm{u}}(T) / R T\right]}
$$

The temperature dependence of $\Delta G_{\mathrm{u}}(T)$ is given by a general Gibbs-Helmholtz relationship (21):

$\Delta G_{\mathrm{u}}(T)=\Delta H_{\mathrm{u}}^{\circ}-\left(\frac{T}{T^{\circ}}\right)\left(\Delta H_{\mathrm{u}}^{\circ}-\Delta G_{\mathrm{u}}^{\circ}\right)-\Delta C_{p, \mathrm{u}}\left[T^{\circ}-T+T \ln \left(\frac{T}{T^{\circ}}\right)\right]$

where $\Delta C_{p, \mathrm{u}}$ is the change in heat capacity upon unfolding, assumed here to be independent of temperature over the range of the experiment $(22) . \Delta G_{\mathrm{u}}^{\circ}$ and $\Delta H_{\mathrm{u}}^{\circ}$ are the free energy and enthalpy of unfolding, respectively, at a reference temperature, $T^{\circ}$. If we set $T^{\circ}$ equal to a $T_{\mathrm{m}}$, then $\Delta G_{\mathrm{u}}^{\circ}$ is equal to zero by definition and the expression becomes

$$
\Delta G_{\mathrm{u}}(T)=\Delta H_{\mathrm{u}}^{\circ}\left(1-\frac{T}{T_{\mathrm{m}}}\right)-\Delta C_{p, \mathrm{u}}\left[T_{\mathrm{m}}-T+T \ln \left(\frac{T}{T_{\mathrm{m}}}\right)\right]
$$

Combining eqs 3 and 5 and rearranging, we obtain

$$
K_{\mathrm{u}}(T)=\mathrm{e}^{-\left\{\frac{\Delta H_{\mathrm{u}}^{\circ}}{R}\left(\frac{1}{T}-\frac{1}{T_{\mathrm{m}}}\right)-\frac{\Delta C_{p}, \mathrm{u}}{R}\left[\frac{T_{\mathrm{m}}}{T}-1+\ln \left(\frac{T}{T_{\mathrm{m}}}\right)\right]\right\}}
$$

and substituting into eq 2

$$
f(T)=\frac{1}{1+\mathrm{e}^{-\left\{\frac{\Delta H_{\mathrm{u}}^{\circ}}{R}\left(\frac{1}{T}-\frac{1}{T_{\mathrm{m}}}\right)-\frac{\Delta C_{p, \mathrm{u}}}{R}\left[\frac{T_{\mathrm{m}}}{T}-1+\ln \left(\frac{T}{T_{\mathrm{m}}}\right)\right]\right\}}}
$$

Proteins undergo unfolding transitions at both low and high temperatures (23) (Figure 1). Although $\Delta G_{\mathrm{u}}(T)$ varies strongly with temperature, $f(T)$ varies significantly only near the $T_{\mathrm{m}}$ values and is otherwise approximately equal to one or zero where the folded or unfolded states, respectively, predominate. At the reference $T_{\mathrm{m}}$ (typically, only the "hot" $T_{\mathrm{m}}$ is experimentally accessible), the term dependent on $\Delta C_{p, \mathrm{u}}$ in eqs $5-7$ is zero and remains 


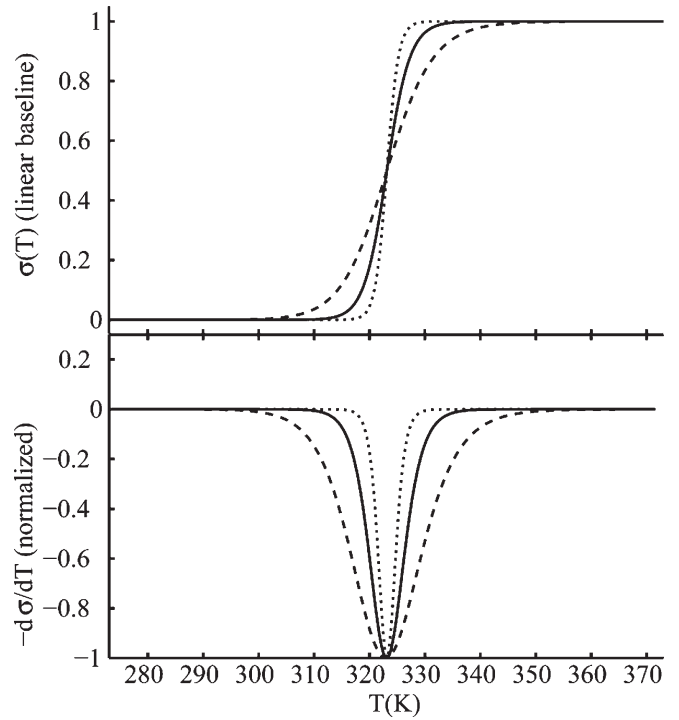

FIGURE 2: Simulation of thermal (un)folding curves incorporating a linear baseline model and illustrating the inverse first derivatives. The top panel shows eq 12 with the following values: $T_{\mathrm{m}}=50^{\circ} \mathrm{C}(323 \mathrm{~K})$, $m_{\mathrm{N}}=0, b_{\mathrm{N}}=0, m_{\mathrm{D}}=0$, and $b_{\mathrm{D}}=1$ and $\Delta H_{\mathrm{u}}$ values of $100(-)$, $200(\cdots)$, and $50 \mathrm{kcal} / \mathrm{mol}(---)$. The bottom panel shows eq 13, the inverse first-derivative transform of the simulation.

negligible in the transition region. Consequently, it is not possible to obtain accurate estimates of $\Delta C_{p, \mathrm{u}}$ from denaturation data obtained at only one of the two transitions. To fit a (un)folding transition around a single transition temperature, we drop the $\Delta C_{p, \mathrm{u}}$ term, which yields the integrated van't Hoff equation (24):

$$
K_{\mathrm{u}}(T)=\mathrm{e}^{-\left[\frac{\Delta H_{\mathrm{u}}^{\circ}}{R}\left(\frac{1}{T}-\frac{1}{T_{\mathrm{m}}}\right)\right]}
$$

Substituting into eq 2:

$$
f(T)=\frac{1}{1+\mathrm{e}^{-\left[\frac{\Delta H_{\mathrm{u}}^{\circ}}{R}\left(\frac{1}{T}-\frac{1}{T_{\mathrm{m}}}\right)\right]}}
$$

Equation 9 is appropriate over the typically narrow temperature ranges in which $f(T)$ transitions between zero and one and, together with eq 1 , provides a straightforward prescription for extracting $\Delta H_{\mathrm{u}}^{\circ}$ and $T_{\mathrm{m}}$ values from experimental data.

In our analysis, we use the inverse first derivative of the experimental data (Figure 2). In this transformation, the transition region manifests itself as a prominent trough, the minimum and width of which are directly related to $T_{\mathrm{m}}$ and $\Delta H_{\mathrm{u}}^{\circ}$ values, respectively (25). The minimum value of the trough is a good initial estimate of $T_{\mathrm{m}}$ for fitting by least-squares minimization [though this minimum may deviate from the $T_{\mathrm{m}}$ with some nonlinear baseline models (see below)]. Differentiating eq 1:

$\frac{\mathrm{d} \sigma}{\mathrm{d} T}=\beta_{\mathrm{N}}\left(\frac{\mathrm{d} f}{\mathrm{~d} T}\right)+\left(\frac{\mathrm{d} \beta_{\mathrm{N}}}{\mathrm{d} T}\right) f(T)-\beta_{\mathrm{D}}\left(\frac{\mathrm{d} f}{\mathrm{~d} T}\right)+\left(\frac{\mathrm{d} \beta_{\mathrm{D}}}{\mathrm{d} T}\right)[1-f(T)]$

and differentiating eq 9:

$$
\frac{\mathrm{d} f}{\mathrm{~d} T}=\left(\frac{-\Delta H_{\mathrm{u}}^{\circ}}{R T^{2}}\right) f(T)[1-f(T)]
$$

Equations 9-11 may be combined with appropriate functional forms of $\beta_{\mathrm{N}}(T)$ and $\beta_{\mathrm{D}}(T)$ to fit the first derivative transform of the experimental data. For example, using linear baseline models $\beta_{\mathrm{N}}(T)=m_{\mathrm{N}} T+b_{\mathrm{N}}$ and $\beta_{\mathrm{D}}(T)=m_{\mathrm{D}} T+b_{\mathrm{D}}$ with eq 1 :

$$
\sigma(T)=\left(m_{\mathrm{N}} T+b_{\mathrm{N}}\right) f(T)+\left(m_{\mathrm{D}} T+b_{\mathrm{D}}\right)[1-f(T)]
$$

This linear baseline model (Figure 2) is appropriate for analysis of experimental data obtained by monitoring the CD signal or tryptophan fluorescence emission intensity (26). In this case, baseline derivatives are $\mathrm{d} \beta_{\mathrm{N}} / \mathrm{d} T=m_{\mathrm{N}}$ and $\mathrm{d} \beta_{\mathrm{D}} /$ $\mathrm{d} T=m_{\mathrm{D}}$, and by combining them with eqs 10 and 11 , we obtain

$$
\begin{aligned}
& \frac{\mathrm{d} \sigma}{\mathrm{d} T}=\left(m_{\mathrm{N}} T+b_{\mathrm{N}}-m_{\mathrm{D}} T-b_{\mathrm{D}}\right)\left\{\left(\frac{-\Delta H_{\mathrm{u}}^{\circ}}{R T^{2}}\right) f(T)[1-f(T)]\right\} \\
& +m_{\mathrm{N}} f+m_{\mathrm{D}}[1-f(T)]
\end{aligned}
$$

In combination with eq 9 , eq 13 can be fit to derivative unfolding data to obtain $T_{\mathrm{m}}, \Delta H_{\mathrm{u}}^{\circ}, m_{\mathrm{D}}, b_{\mathrm{D}}, m_{\mathrm{N}}$, and $b_{\mathrm{D}}$. For analysis of $\mathrm{SO}$ data, a nonlinear model is used (see below).

Ligand-Induced Shifts in Protein Stability. The linked equilibrium between single-site ligand binding and protein (un)folding can be written as a thermodynamic cycle:

Scheme 1

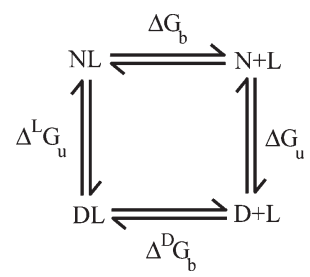

where $\mathrm{N}$ is the folded (native) protein, $\mathrm{D}$ the unfolded (denatured) protein, L the ligand, NL the native proteinligand complex, and DL the denatured protein-ligand complex. We use the convention that positive free energy of unfolding $\left(\Delta G_{\mathrm{u}}\right)$ values favor the folded state and positive free energy of binding $\left(\Delta G_{\mathrm{b}}\right)$ values favor the unbound state. This cycle can be written as

$$
\Delta G_{\mathrm{u}}(T)-\Delta G_{\mathrm{b}}\left([\mathrm{L}]_{\mathrm{T}}, T\right)-\Delta^{L} G_{\mathrm{u}}\left([\mathrm{L}]_{\mathrm{T}}, T\right)+\Delta^{\mathrm{D}} G_{\mathrm{b}}\left([\mathrm{L}]_{\mathrm{T}}, T\right)=0
$$

where $[\mathrm{L}]_{\mathrm{T}}$ is the total ligand concentration and $\mathrm{T}$ the absolute temperature. A rearrangement shows the relationship between stability change and binding:

$$
\Delta G_{\mathrm{u}}(T)-\Delta^{L} G_{\mathrm{u}}\left([\mathrm{L}]_{\mathrm{T}}, T\right)=\Delta G_{\mathrm{b}}\left([\mathrm{L}]_{\mathrm{T}}, T\right)-\Delta^{D} G_{\mathrm{b}}\left([\mathrm{L}]_{\mathrm{T}}, T\right)
$$

abbreviated as

$$
\Delta \Delta G_{\mathrm{u}}\left([\mathrm{L}]_{\mathrm{T}}, T\right)=\Delta \Delta G_{\mathrm{b}}\left([\mathrm{L}]_{\mathrm{T}}, T\right)
$$

The change in protein stability due to ligand binding is equivalent to the free energy difference between the ligand binding to the native state and to the denatured state. If the ligand does not bind to the denatured state (which is often the case), the observed stability increase is equivalent to the free energy of binding to the native state.

The free energy of binding can be expressed as a function of ligand with the canonical equation of macromolecular binding $(27,28)$ :

$$
\Delta G_{\mathrm{b}}=-R T \ln Q
$$


where $Q$ is the binding polynomial (29) for binding of the native protein to ligands. Substituting eq 17 into eq 16

$$
\begin{aligned}
\Delta \Delta G_{\mathrm{u}}\left([\mathrm{L}]_{\mathrm{T}}, T\right) & =\Delta \Delta G_{\mathrm{b}}\left([\mathrm{L}]_{\mathrm{T}}, T\right) \\
& =\Delta G_{\mathrm{b}}\left([\mathrm{L}]_{\mathrm{T}}, T\right)-\Delta^{D} G_{\mathrm{b}}\left([\mathrm{L}]_{\mathrm{T}}, T\right) \\
& =-R T \ln Q+R T \ln ^{D} Q \\
& =-R T \ln \frac{Q}{D} Q
\end{aligned}
$$

where ${ }^{\mathrm{D}} Q$ is the binding polynomial for binding of the denatured protein to ligands. The binding polynomial is the sum of concentrations of all states of the protein relative to free protein (i.e., the partition function). For a single ligand binding to a single site

$$
\begin{gathered}
Q=\frac{[\mathrm{N}]}{[\mathrm{N}]}+\frac{[\mathrm{NL}]}{[\mathrm{N}]}=1+\frac{[\mathrm{L}]}{K_{\mathrm{d}}} \\
{ }^{D} Q=\frac{[\mathrm{D}]}{[\mathrm{D}]}+\frac{[\mathrm{DL}]}{[\mathrm{D}]}=1+\frac{[\mathrm{L}]}{{ }^{D} K_{\mathrm{d}}}
\end{gathered}
$$

Consequently, if a single ligand binds to a single site of both the native and denatured states:

$$
\Delta \Delta G_{\mathrm{u}}\left([\mathrm{L}]_{\text {free }}, T\right)=-R T \ln \left[\frac{1+\frac{\left[\mathrm{L}_{\text {free }}\right.}{K_{\mathrm{d}}(T)}}{1+\frac{[\mathrm{L}]_{\text {free }}}{D_{\mathrm{d}}(T)}}\right]
$$

where $[\mathrm{L}]_{\text {free }}$ is the concentration of unbound ligand free in solution and $K_{\mathrm{d}}(T)$ and ${ }^{\mathrm{D}} K_{\mathrm{d}}(T)$ are the temperature-dependent equilibrium constants of ligand dissociation with native and denatured protein, respectively.

Three different experimental regimes need to be considered in the use of eq 21 (Figure 3). First, we analyze the case in which both the native and denatured states have appreciable affinity for the ligand (30). The ligand-dependent change in stability is a balance of opposing stabilization of the native and denatured states by the ligand (eq 18). Consequently (if ${ }^{\mathrm{D}} K_{\mathrm{d}}>K_{\mathrm{d}}$, i.e., the denatured state binds more weakly than the native state) at lower ligand concentrations where binding to the native state dominates, a ligand-dependent increase in stability is observed. However, at elevated ligand concentrations where the ligand binds appreciably to the denatured state, the opposing effects lead to "saturation" of the observed shift in stability (Figure 3A).

In the second case, the denatured state has no affinity for ligand. Consequently, ${ }^{\mathrm{D}} K_{\mathrm{d}}$ tends to infinity and the denominator in eq 21 tends to 1 :

$$
\Delta \Delta G_{\mathrm{u}}\left([\mathrm{L}]_{\text {free }}, T\right)=-R T \ln \left[1+\frac{[\mathrm{L}]_{\text {free }}}{K_{\mathrm{d}}(T)}\right]
$$

In this case, stability increases without saturation at progressively higher ligand concentrations (Figure 3B), a behavior that is often observed experimentally.

The third regime takes into consideration the relationship between total ligand and protein concentrations. Equations 21 and 22 describe the thermodynamic relationship in terms of free, not total, ligand concentration and may be used to fit experimental data if ligand concentrations are at least $\sim 1$ order of magnitude in excess over protein $\left([\mathrm{L}]_{\mathrm{T}} \gg[\mathrm{P}]_{\mathrm{T}}\right)$ and $[\mathrm{L}]_{\mathrm{T}} \cong[\mathrm{L}]_{\text {free }}$, where $[\mathrm{L}]_{\mathrm{T}}$ is the total concentration of ligand
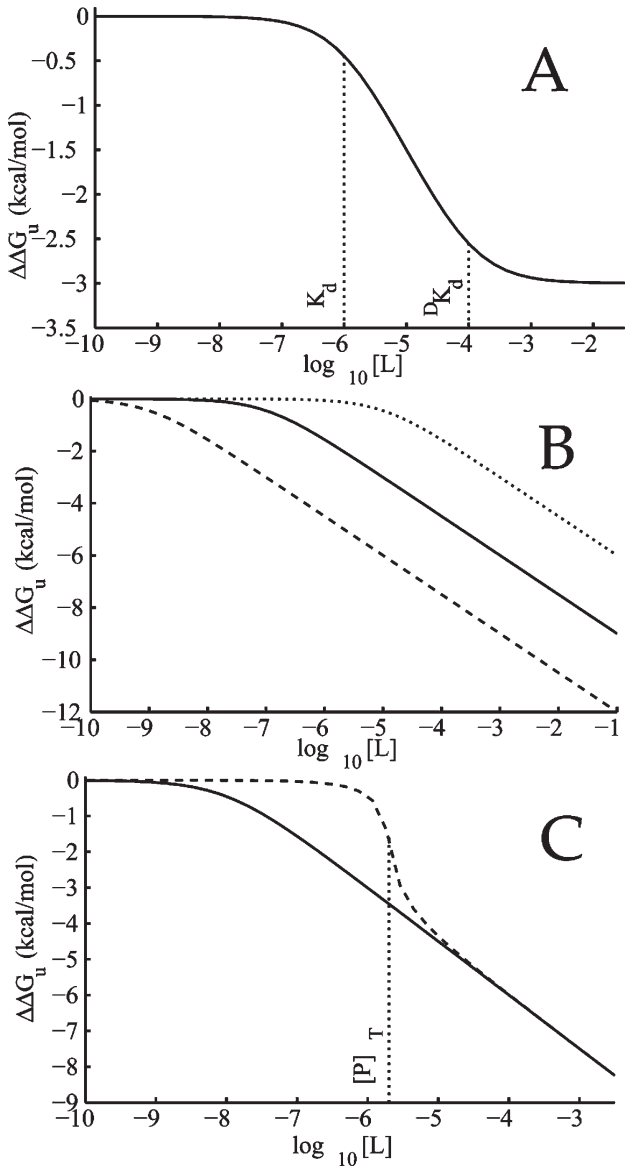

FIgure 3: Three experimental regimes that may be observed in a thermal shift assay. (A) Saturation of the $\Delta \Delta G_{\mathrm{u}}$ shift if the ligand has significant affinity for the denatured state (simulated with eq 21 , $K_{\mathrm{d}}=1 \mu \mathrm{M}$, and ${ }^{\mathrm{D}} K_{\mathrm{d}}=100 \mu \mathrm{M}$ ). (B) Shift in $\Delta \Delta G_{\mathrm{u}}$, if the ligand has no affinity for the denatured state at different $K_{\mathrm{d}}$ values [simulated with eq $22, K_{\mathrm{d}}=1 \mathrm{nM}(--), K_{\mathrm{d}}=100 \mathrm{nM}(-)$, and $K_{\mathrm{d}}=10 \mu \mathrm{M}$ $(\cdots)$ ]. (C) Stoichiometric effects at ligand concentrations near or below the protein concentration [simulated with eq $23, K_{\mathrm{d}}=1 \mathrm{nM}$, and $\left.[\mathrm{P}]_{\mathrm{T}}=2 \mu \mathrm{M}(---)\right]$ and nonstoichiometric [simulated with eq 22 , $\left.K_{\mathrm{d}}=1 \mathrm{nM}(-)\right]$. Equations 22 and 23 are functionally equivalent at ligand concentrations at least 1 order of magnitude above the protein concentration.

$\left([\mathrm{L}]_{\mathrm{T}}=[\mathrm{L}]_{\mathrm{free}}+[\mathrm{NL}]+[\mathrm{DL}]\right)$ and $[\mathrm{P}]_{\mathrm{T}}$ is the total protein concentration $\left([\mathrm{P}]_{\mathrm{T}}=[\mathrm{D}]+[\mathrm{DL}]+[\mathrm{N}]+[\mathrm{NL}]\right)$. In the case in which there is no affinity of ligand for the denatured state $([D L]=0)$, if $[\mathrm{L}]_{\mathrm{T}}$ is less than or in the neighborhood of $[\mathrm{P}]_{\mathrm{T}}$ (the stoichiometric regime), then eq 22 may be expressed in terms of $[\mathrm{L}]_{\mathrm{T}}$ and $[\mathrm{P}]_{\mathrm{T}}$ (see the Supporting Information for derivation):

$$
\begin{gathered}
\Delta \Delta G_{\mathrm{u}}\left([\mathrm{L}]_{\mathrm{T}},[\mathrm{P}]_{\mathrm{T}}, T\right)=-R T \ln \left(1+\left\{[\mathrm{L}]_{\mathrm{T}}-[\mathrm{P}]_{\mathrm{T}}-2 K_{\mathrm{d}}(T)\right.\right. \\
\left.\left.+\sqrt{\left[[\mathrm{L}]_{\mathrm{T}}+[\mathrm{P}]_{\mathrm{T}}+2 K_{\mathrm{d}}(T)\right]^{2}-4[\mathrm{P}]_{\mathrm{T}}[\mathrm{L}]_{\mathrm{T}}}\right\} /\left[2 K_{\mathrm{d}}(T)\right]\right)
\end{gathered}
$$

Under these conditions, the ligand dependence of $\Delta G_{\mathrm{u}}$ deviates from case 2 at low concentrations where protein is in stoichiometric excess over ligand but rejoins at higher ligand concentrations (Figure 3C).

Analysis of thermal denaturations at different ligand concentrations requires that all free energies be referenced to a common temperature. The $T_{\mathrm{m}}$ of the apoprotein $\left({ }^{\mathrm{apo}} T_{\mathrm{m}}\right)$ is a particularly 


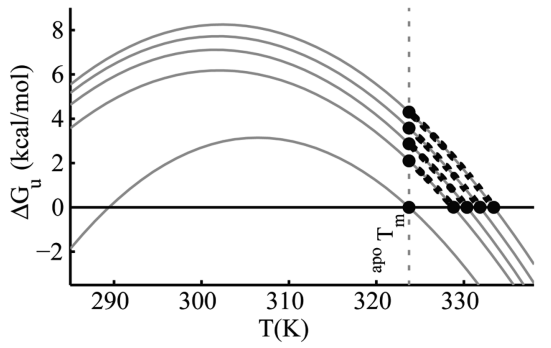

FIgURE 4: Experimental MBP $T_{\mathrm{m}}$ values determined at various maltose concentrations $(0,60,221,814$, and $3 \mu \mathrm{M})$ extrapolated to ligand-induced $\Delta \Delta G_{\mathrm{u}}$ values at the ${ }^{\text {apo }} T_{\mathrm{m}}$ with eq 24 (dotted black lines). Fits to the Gibbs - Helmholtz equation (eq 5 ) are shown in gray for reference. A calculated (34) $\Delta C_{p, \mathrm{u}}$ of $6.5 \mathrm{kcal} \mathrm{mol}^{-1} \mathrm{~K}^{-1}$ was used for the apoprotein and an experimentally estimated $\Delta^{\mathrm{L}} C_{p, \mathrm{u}}$ of $5.5 \mathrm{kcal}$ $\mathrm{mol}^{-1} \mathrm{~K}^{-1}$ for maltose-shifted MBP curves (Figure 13A). Note that these extrapolated $\Delta \Delta G_{\mathrm{u}}$ data are fit to eq 25 to yield $K_{\mathrm{d}}$ in Figure 13B. Data were obtained with $20 \times \mathrm{SO}$.

appropriate reference point because $\Delta G_{\mathrm{u}}$ and $\Delta \Delta G_{\mathrm{u}}$ equal zero. From the Gibbs-Helmholtz relationship (eq 5), the stability difference between apo and liganded protein is

$$
\begin{gathered}
\Delta \Delta G_{\mathrm{u}}\left([\mathrm{L}]_{\mathrm{T}}\right)=-\Delta^{L} H_{\mathrm{u}}\left(1-\frac{{ }^{a p o} T_{\mathrm{m}}}{{ }^{L} T_{\mathrm{m}}}\right) \\
+\Delta^{L} C_{p, \mathrm{u}}\left[{ }^{L} T_{\mathrm{m}}-{ }^{a p o} T_{\mathrm{m}}+{ }^{a p o} T_{\mathrm{m}} \ln \left(\frac{{ }^{a p o} T_{\mathrm{m}}}{{ }^{L} T_{\mathrm{m}}}\right)\right]
\end{gathered}
$$

where ${ }^{\mathrm{L}} T_{\mathrm{m}}, \Delta \Delta^{\mathrm{L}} H_{\mathrm{u}}$, and $\Delta^{\mathrm{L}} C_{p, \mathrm{u}}$ are the $T_{\mathrm{m}}$, enthalpy, and heat capacity of unfolding, respectively, of the protein at ligand concentration $[\mathrm{L}]_{\mathrm{T}}$. This equation is used to calculate $\Delta \Delta G_{\mathrm{u}}$ at $[\mathrm{L}]_{\mathrm{T}}$ by extrapolation from the various experimentally determined ${ }^{\mathrm{L}} T_{\mathrm{m}}$ values to the common reference ${ }^{\text {apo }} T_{\mathrm{m}}$ (Figure 4) using $\Delta^{\mathrm{L}} C_{p, \mathrm{u}}$ (see below) and $\Delta^{\mathrm{L}} H_{\mathrm{u}}$ (from experiment).

Because ligand-dependent changes in stability are all extrapolated to the common ${ }^{\text {apo }} T_{\mathrm{m}}$, the relationship between folding and binding free energies (eq 22) becomes

$$
\Delta \Delta G_{\mathrm{u}}^{\circ}\left([\mathrm{L}]_{\text {free }}\right)=-R^{a p o} T_{\mathrm{m}} \ln \left(1+\frac{[\mathrm{L}]_{\text {free }}}{K_{\mathrm{d}}^{\circ}}\right)
$$

where $K_{\mathrm{d}}^{\circ}$ is the $K_{\mathrm{d}}$ and $\Delta \Delta G_{\mathrm{u}}^{\circ}$ the extrapolated stability free energy change at the reference temperature. Or in the stoichiometric regime (eq 23)

$$
\begin{gathered}
\Delta \Delta G_{\mathrm{u}}^{\circ}\left([\mathrm{L}]_{\mathrm{T}},[\mathrm{P}]_{\mathrm{T}}\right)=-R^{a p o} T_{\mathrm{m}} \ln \left\{1+\left[[\mathrm{L}]_{\mathrm{T}}-[\mathrm{P}]_{\mathrm{T}}-2 K_{\mathrm{d}}^{\circ}\right.\right. \\
\left.\left.+\sqrt{\left([\mathrm{L}]_{\mathrm{T}}+[\mathrm{P}]_{\mathrm{T}}+2 K_{\mathrm{d}}^{\circ}\right)^{2}-4[\mathrm{P}]_{\mathrm{T}}[\mathrm{L}]_{\mathrm{T}}}\right] /\left(2 K_{\mathrm{d}}^{\circ}\right)\right\}
\end{gathered}
$$

In this analysis, experimentally determined ligand affinities are therefore reported at the ${ }^{\text {apo }} T_{\mathrm{m}}$.

Heat Capacity of Unfolding. By definition, the heat capacity of unfolding, $\Delta C_{p, \mathrm{u}}$, equals $\mathrm{d} \Delta H_{\mathrm{u}} / \mathrm{d} T$. Its value can be estimated experimentally by systematic variation of $T_{\mathrm{m}}$ and observation of the corresponding $\Delta H_{\mathrm{u}}(21,31-33)$, which in the case of measuring ligand interactions is obtained naturally in a titration series. In addition to modulating the temperatures ( $T_{\mathrm{m}}$ values) at which observations of $\Delta H_{\mathrm{u}}$ are made, ligand binding itself causes a change in the observed enthalpy of unfolding, $\Delta^{\text {obs }} H_{\mathrm{u}}$. We consider the case in which ligand has no affinity for the denatured state. As the protein binds ligand, $\Delta^{\text {obs }} H_{\mathrm{u}}$ undergoes a transition from being the enthalpy difference between $\mathrm{N}$ and $\mathrm{D}\left(\Delta H_{\mathrm{u}}\right)$ to being

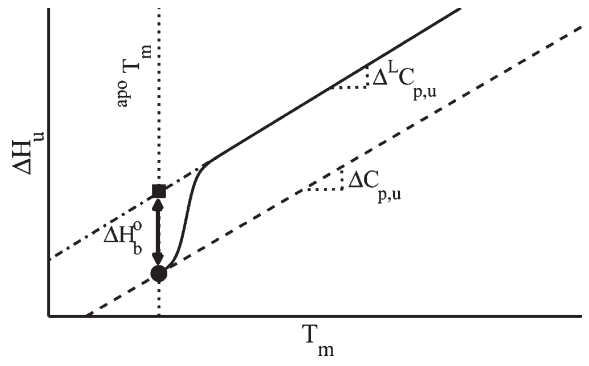

FIGURE 5: Observed variation of $\Delta H_{\mathrm{u}}$ with ligand-induced stability shifts [eq $27(-)$ ]. The observed shift has two components: (1) initial transition with the enthalpy of binding, $\Delta H_{\mathrm{b}}$, proportional to the fraction bound and (2) linear temperature dependence of the liganded protein $\left[\Delta^{\mathrm{L}} H_{\mathrm{u}}=\Delta^{\mathrm{L}} H_{\mathrm{u}}^{\circ}+\Delta^{L} C_{p, u}\left(T_{m}-{ }^{a p o} T_{m}\right)\right]$. Included for reference are the $\Delta H_{u}$ of the apoprotein (---), the $\Delta^{L} H_{u}$ of the liganded protein $(\cdot \cdots), \Delta H_{u}^{\circ}(\bullet)$, and $\Delta^{L} H_{u}^{\circ}(\mathbf{\square})$.

the enthalpy difference between NL and $\mathrm{D}+\mathrm{L}\left(\Delta^{\mathrm{L}} H_{\mathrm{u}}\right)$. The difference between $\Delta H_{\mathrm{u}}$ and $\Delta^{\mathrm{L}} H_{\mathrm{u}}$ is the enthalpy of binding $\left(\Delta H_{\mathrm{b}}\right)$. Ligand binding therefore changes the value of $\Delta^{\mathrm{obs}} H_{\mathrm{u}}$ proportionally to the fraction of protein bound by ligand (Figure 5). Consequently, the temperature dependence of $\Delta^{\text {obs }} H_{\mathrm{u}}$ undergoes a transition from a line with a slope $\Delta C_{p, \mathrm{u}}$ for the apoprotein ( $\mathrm{N}$ to $\mathrm{D}$ ) to a line with slope $\Delta^{\mathrm{L}} C_{p, \mathrm{u}}$ for the ligand-bound form (NL to D $+\mathrm{L}$ ), as described by

$$
\begin{aligned}
& \Delta^{o b s} H_{\mathrm{u}}(\left.T_{\mathrm{m}},[\mathrm{L}]_{\text {free }}\right)=\left(\frac{K_{\mathrm{d}}}{K_{\mathrm{d}}+[\mathrm{L}]_{\text {free }}}\right)\left[\Delta H_{\mathrm{u}}^{\circ}+\Delta C_{p, \mathrm{u}}\left(T_{\mathrm{m}}-{ }^{a p o} T_{\mathrm{m}}\right)\right] \\
&+\left(\frac{[\mathrm{L}]_{\text {free }}}{K_{\mathrm{d}}+[\mathrm{L}]_{\text {free }}}\right)\left[\Delta^{L} H_{\mathrm{u}}^{\circ}+\Delta^{L} C_{p, \mathrm{u}}\left(T_{\mathrm{m}}-{ }^{a p o} T_{\mathrm{m}}\right)\right]
\end{aligned}
$$

where $\Delta^{\mathrm{L}} H_{\mathrm{u}}^{\mathrm{o}}$ is a notional quantity equal to the enthalpy of unfolding of the ligand-bound form extrapolated back to the ${ }^{\text {apo }} T_{\mathrm{m}}$ (see Figure 5). The enthalpy of binding is

$$
\Delta H_{\mathrm{b}}^{\circ}=\Delta H_{\mathrm{u}}^{\circ}-\Delta^{L} H_{\mathrm{u}}^{\circ}
$$

and the heat capacity of binding

$$
\Delta C_{p, \mathrm{~b}}=\Delta C_{p, \mathrm{u}}-\Delta^{L} C_{p, \mathrm{u}}
$$

In practice, the dependence of $\Delta^{\mathrm{L}} H_{\mathrm{u}}$ on $T_{\mathrm{m}}$ for a series of ligand concentrations is fit to a line with slope $\Delta^{\mathrm{L}} C_{p, \mathrm{u}}$. Alternatively, $\Delta^{\mathrm{L}} C_{p, \mathrm{u}}$ can be estimated from the size of the protein (34) if it is assumed that $\Delta^{\mathrm{L}} C_{p, \mathrm{u}} \cong \Delta C_{p, \mathrm{u}}$.

Monitoring Protein Folding with Sypro Orange. Sypro Orange (SO) (14) is a member of a family of fluorescent dyes (35) that are highly sensitive to their environment. SO fluorescence emission intensity (FEI) exhibits strong solvent effects, a nonlinear temperature dependence (Figure 6), and increases in the presence of denatured protein. A typical protein thermal denaturation monitored by SO, showing stability shifts upon addition of ligand or denaturant, is shown in Figure 7. The post-transition baseline exhibits a pronounced, nonlinear decrease in FEI with temperature (Figure 8A). This decrease in signal has been attributed primarily to protein aggregation (13) and irreversible interaction of SO with the denatured protein (3). For MBP, we find that the unfolding transition is quite reversible (Figure $8 \mathrm{~B}$ ) and that signal reversibility is maintained at temperatures where the unfolded state is highly populated in the early downward-sloping post-transition region (Figure 8C). The nonlinear temperature dependence in this early region for this particular protein therefore presumably 


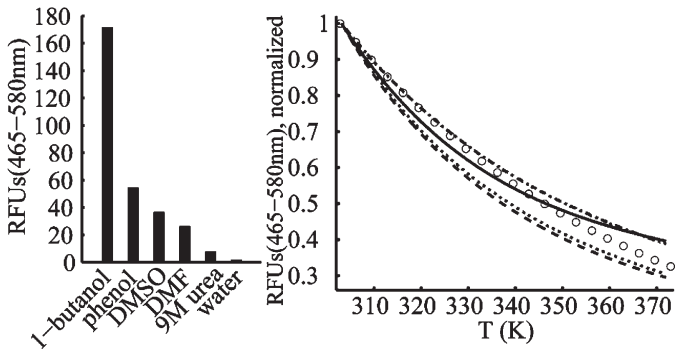

FiguRE 6: Fluorescence emission intensities $(465-580 \mathrm{~nm})$ of $20 \times$ Sypro Orange in various solvents. Comparison of emission intensities at $30{ }^{\circ} \mathrm{C}$ (left). Temperature dependence of normalized emission intensities (right): 1-butanol (-), phenol (saturated with Tris-EDTA buffer) (---), DMSO $(\cdots)$, DMF (*-•), and normalized exponential decay curve $\exp (-0.016 \times T)$ shown for comparison $(O)$.

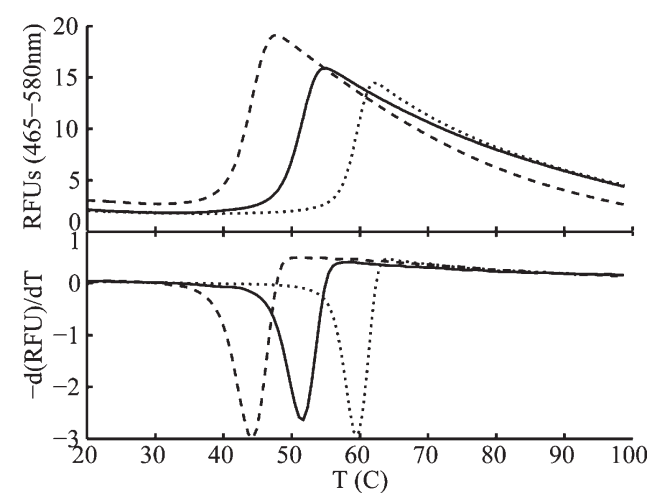

FIGURE 7: Experimental thermal melts of MBP (top) and corresponding inverse derivatives (bottom) measured for the apoprotein $(-)$, in the presence of ligand $(0.3 \mathrm{mM}$ maltose $)(\cdots)$, or in the presence of denaturant (1.4 M urea) (---). All melts were determined in the presence of $20 \times$ Sypro Orange.

reflects an intrinsic property of dye photophysics, consistent with the temperature dependence of SO fluorescence in low-dielectric solvents (Figure 6). At increasing temperatures, however, irreversible aggregation processes could make increasing contributions to the post-transition baseline signal. Even so, limitations on reversibility are not intrinsic to the use of the dye, and at least for some proteins, the characteristic downward-sloping nonlinear baseline observed in SO-monitored protein melts has a greater reversible contribution from dye photophysics than may have been appreciated previously.

We fit this post-transition baseline with a nonlinear, empirically derived single exponential. This approach is based on the premise that the system continues to be in equilibrium through the post-transition temperature range. An alternative approach is to crop the data at the post-transition region because the system it is not in equilibrium at these elevated temperatures. This approach generally implies a linear baseline with a constant value. The choice of baseline affects the extracted $T_{\mathrm{m}}$ value and may introduce systematic error. The correct choice of baseline will be dependent on the reversibility of the system. In this study, we favor the nonlinear exponential because it fits the experimental data well (Figure 9) and because of the nonlinear temperature dependence of dye FEI in solvent (Figure 6).

To analyze protein denaturation of $\mathrm{MBP}, \mathrm{SN}$, and $\mathrm{RBP}$ monitored with SO, we use an exponential function, $A \mathrm{e}^{-\lambda T}$, to describe the nonlinear post-transition baseline $\left(\beta_{\mathrm{D}}\right)$ and a constant, $B$, to describe the pretransition baseline $\left(\beta_{\mathrm{N}}\right)$. The fluorescence
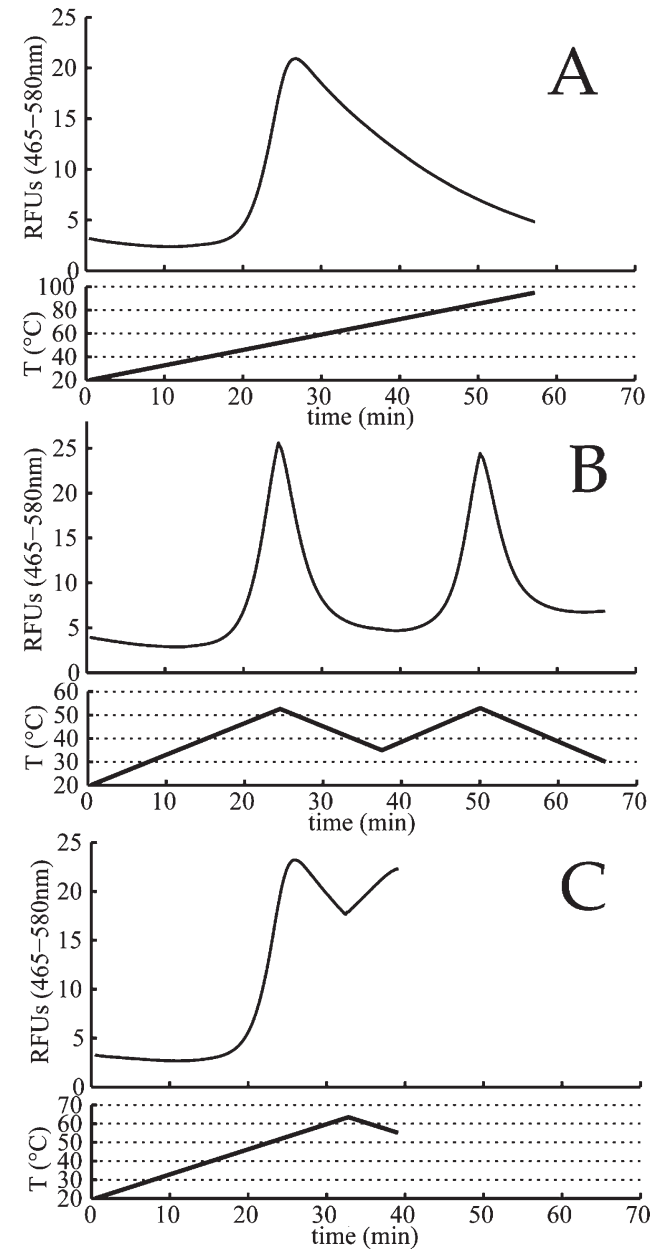

FiguRE 8: Reversibility of thermal melts of MBP monitored with SO. (A) Forward denaturation curve (inset showing the time dependence of temperature change, ramp rate of $1.3{ }^{\circ} \mathrm{C} / \mathrm{min}$ ). (B) Reversibility of the transition region. The temperature is taken to nearmaximal fluorescence intensity (cf. panel A), decreased (inset), increased again, and decreased again. Note the similarity of the two transitions indicating a high degree of reversibility. (C) Reversibility in the post-transition region. The system is heated to a temperature where the unfolded state is well-populated, at which point it is cooled to the temperature of maximal fluorescence (inset). Note the nearly full recovery of fluorescence intensity, consistent with the intrinsic temperature dependence of dye fluorescence.

signal of two-state denaturation monitored with $\mathrm{SO}$ is then (from eq 1)

$$
\sigma(T)=B f(T)+A \mathrm{e}^{-\lambda T}[1-f(T)]
$$

Applying eqs 10 and 11

$$
\begin{aligned}
\frac{\mathrm{d} \sigma}{\mathrm{d} T}=\left\{B\left(\frac{-\Delta H_{\mathrm{u}}^{\circ}}{R T^{2}}\right) f(T)[1-f(T)]\right\} \\
-\left\{A \mathrm{e}^{-\lambda T}[1-f(T)]\left[\frac{-\Delta H_{\mathrm{u}}^{\circ}}{R T^{2}} f(T)+\lambda\right]\right\}
\end{aligned}
$$

Equation 31 (with eqs 9 and 11) can be fit to inverse derivative data (in this case exported directly from an RT-PCR instrument) to yield $\Delta H_{\mathrm{u}}^{\circ}$ and $T_{\mathrm{m}}$ (see the Supporting Information for more details). Representative fits to experimental data are given in Figure 9. Note that at high values of $\lambda, T_{\mathrm{m}}$ deviates from the minimum value of the derivative trough because of the nonlinear character of $\beta_{\mathrm{D}}$ (Figure 9C). 

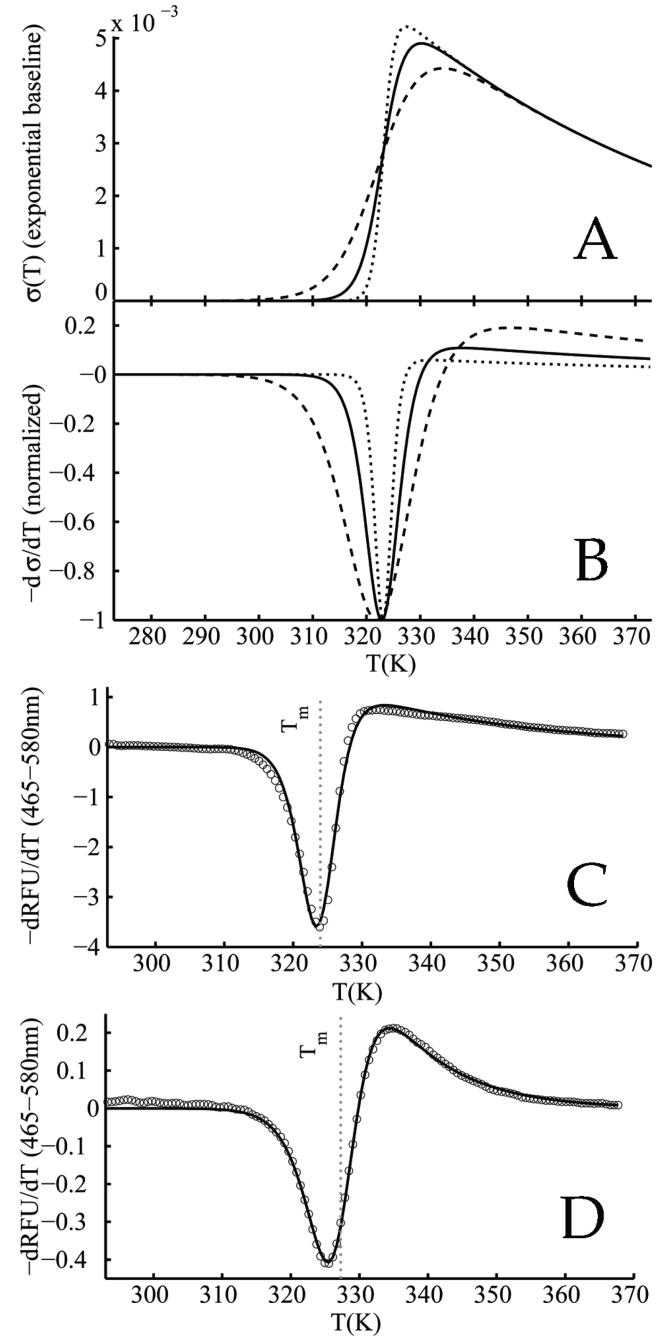

Figure 9: Exponential baseline model illustrated with simulated (A and B) and experimental (C and D) thermal melts. (A) Simulations (eq 30) of thermal melts $\left(T_{\mathrm{m}}=323 \mathrm{~K}, A=1, B=0\right.$, and $\lambda=0.016$ ) illustrating the effect of $\Delta H_{\mathrm{u}}[100(-), 200(\cdots)$, and $50 \mathrm{kcal} / \mathrm{mol}(--)]$. (B) Inverse first derivatives (eq 31) with the same values as in panel A. (C) Experimental melting data for MBP $(O)$ fit to eq $31(-)\left(T_{\mathrm{m}}=\right.$ $51^{\circ} \mathrm{C}, \Delta H_{\mathrm{u}}=116 \mathrm{kcal} / \mathrm{mol}$, and $\left.\lambda=0.041\right)$. (D) Experimental melting data for $\mathrm{SN}(\mathrm{O})$ fit to eq $31(-)\left(T_{\mathrm{m}}=54^{\circ} \mathrm{C}, \Delta H_{\mathrm{u}}=95 \mathrm{kcal} / \mathrm{mol}\right.$, and $\lambda=0.103)$. Data were collected at $20 \times \mathrm{SO}$. Note that at high $\lambda$ values the denatured baseline is highly nonlinear and the $T_{\mathrm{m}}$ is shifted off of the inflection point.

Rate of Temperature Change. The thermodynamic analysis described above implies that experimental observations are made under equilibrium conditions. The thermal denaturations are conducted by progressively increasing ("ramping") the temperature while simultaneously observing a change in fluorescence. The rate of temperature change must therefore allow the relaxation of all processes to equilibrium given the kinetics of folding, binding, interaction with $\mathrm{SO}$, and thermal equilibration over the sample volume. Modern RT-PCR instruments can quickly ramp the temperature and may outstrip equilibration. To test whether equilibrium is established at a given ramp rate, a temperature in the transition region was held constant for a period of time and the subsequent change in fluorescence observed. For MBP at a ramp rate of $5.6{ }^{\circ} \mathrm{C} / \mathrm{min}$, several seconds was required to reach equilibrium (Figure 10A), whereas when the rate was $1.3{ }^{\circ} \mathrm{C} / \mathrm{min}$, equilibration was almost instantaneous (Figure 10B).
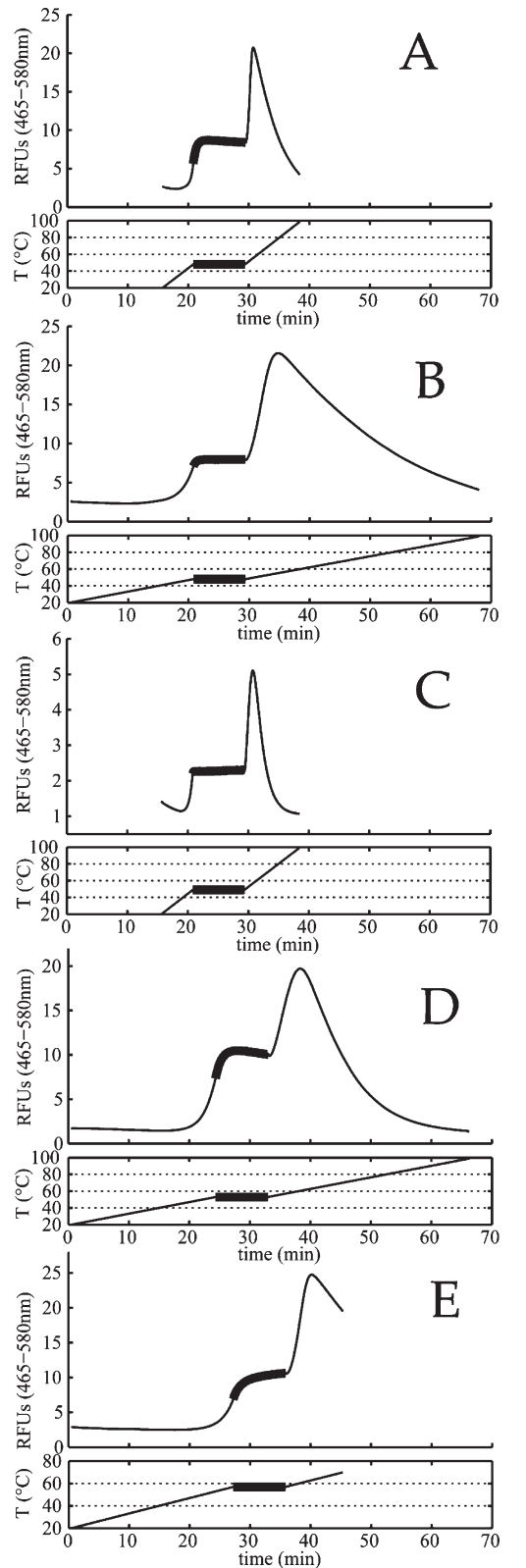

FIGURE 10: Effect of temperature ramp rates on equilibration. (A) At $5.6{ }^{\circ} \mathrm{C} / \mathrm{min}$, the temperature ramp rate exceeds equilibration conditions for MBP. The temperature is ramped up to the approximate $T_{\mathrm{m}}$ value, held constant while isothermal changes in FEI are monitored, and then ramped to well beyond the transition region (inset). A significant change in fluorescence intensity emission during the isothermal dwell period (thick black line) indicates that the system is not in equilibrium. (B) At $1.3{ }^{\circ} \mathrm{C} / \mathrm{min}, \mathrm{MBP}$ remains approximately at equilibrium during the temperature changes. (C) At $5.6^{\circ} \mathrm{C} / \mathrm{min}$, $\mathrm{SN}$ remains approximately at equilibrium during the temperature changes. (D) At $1.3^{\circ} \mathrm{C} / \mathrm{min}, \mathrm{RBP}$ is not at equilibrium. (E) Addition of $3 \mathrm{mM}$ maltose alters the equilibration conditions of MBP at a ramp rate of $1.3^{\circ} \mathrm{C} / \mathrm{min}$ (cf. panel B) as a consequence of additional linked equilibria.

SO Concentration Effects. In the absence of any protein, the FEI of SO in aqueous buffer peaks at a critical SO concentration (Figure 11), consistent with self-association of SO (where the self-associated dye is quenched relative to the monomeric dye in solution), a phenomenon commonly observed with aromatic small molecule dyes (36) (Figure 11B). If $\mathrm{SO}$ self-associates and is autoquenched, changes in FEI upon interaction with denatured protein could have two origins: a "passive" effect in which a decrease in the free 

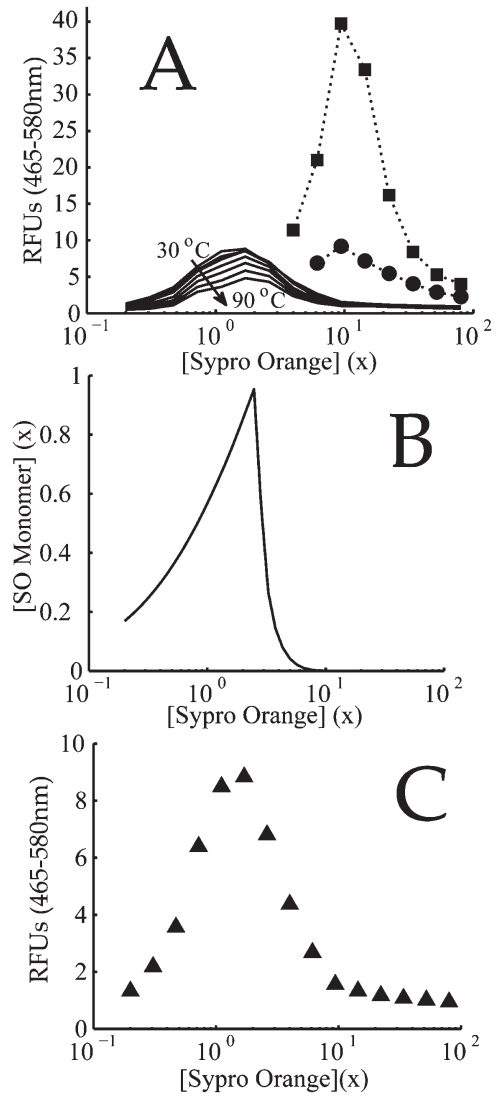

Figure 11: Effects of Sypro Orange concentration. (A) Concentration dependence of Sypro Orange (SO) fluorescence emission intensity in the absence $[25 \mathrm{mM}$ potassium phosphate $(\mathrm{pH} 7.5), 140 \mathrm{mM}$ $\mathrm{KCl}$, from 30 to $90{ }^{\circ} \mathrm{C}$ in $10{ }^{\circ} \mathrm{C}$ increments (一)] and presence of protein [peak fluorescence of the transition of melts of MBP $(\square)$ and SN (@) at all SO concentrations that yielded a clear melting transition]. A qualitatively similar SO fluorescence peak is observed in the absence of protein when constant $1.4 \%$ DMSO cosolvent is maintained across $\mathrm{SO}$ concentrations (data not shown). (B) An attenuated $K$ model of indefinite self-association (36) qualitatively accounts for the concentration dependence of the SO fluorescence emission intensity (simulated with $\tau=1$ and $K_{\mathrm{A}}=1 \times$; see eq 19 in ref 36 for definition of symbols; SO concentrations are given in arbitrary units $\times$ ). (C) Experimental SO fluorescence emission intensity at $30{ }^{\circ} \mathrm{C}(\mathbf{\Delta})$, shown for comparison with the qualitative model in panel B.

SO concentration ${ }^{2}$ shifts the self-association equilibrium toward the more fluorescent free monomer or an "active" effect in which FEI increases through direct interaction with the denatured state. For SN denaturation, the peak signal is comparable in intensity to the FEI peak of free SO in buffer (Figure 11A) and could be ascribed to a passive effect, whereas the MBP transition shows a significantly higher FEI peak that can be ascribed to only an active interaction with the protein.

Interaction of $\mathrm{SO}$ with the denatured state is expected to lower the $T_{\mathrm{m}}$ with an increased dye concentration. The apparent $T_{\mathrm{m}}$ values of SN remain nearly constant over a 1 order of magnitude change in SO concentration, whereas a $\sim 6{ }^{\circ} \mathrm{C}$ decrease is observed for MBP over the same concentration range. The ligand affinities of MBP (Figure 12B) exhibit a concomitant dependence on $\mathrm{SO}$ concentration, but for $\mathrm{SN}$, these remain nearly constant

${ }^{2}$ Sypro Orange concentrations are given in arbitrary units $\times$ provided by the manufacturer because the molar extinction coefficient is not publicly available. In the absence of absolute concentrations, we did not develop a more quantitative model.

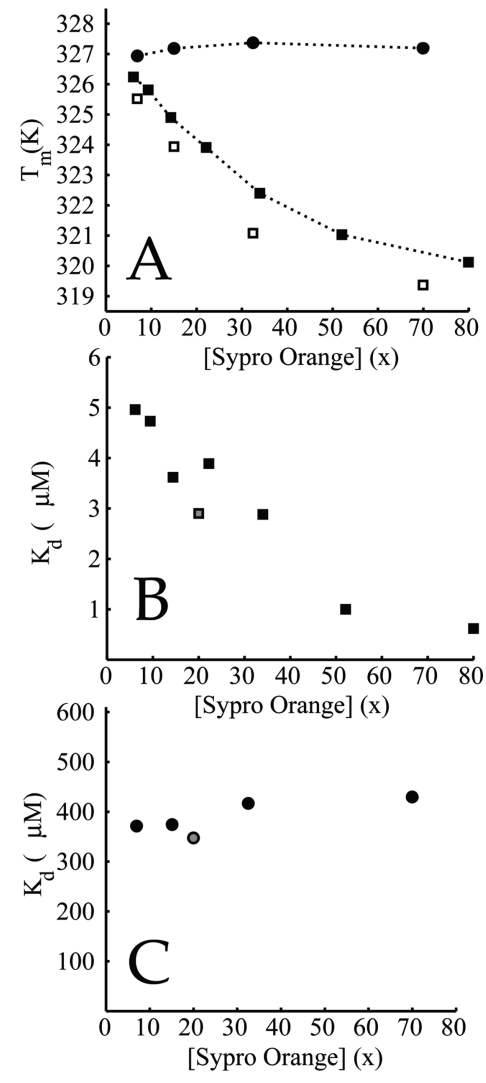

Figure 12: Variation of $T_{\mathrm{m}}$ and $K_{\mathrm{d}}$ values with Sypro Orange (SO) concentration. (A) Apparent $T_{\mathrm{m}}$ of SN () and MBP [(ם) DMSO variable with SO from 0.1 to $1.6 \%$ and $(\square)$ constant $1.4 \%$ DMSO cosolvent]. (B) Apparent $K_{\mathrm{d}}$ of MBP for maltose as determined from melts at $0 \mathrm{mM}, 100 \mu \mathrm{M}$, and $1 \mathrm{mM}$ maltose $(\mathbb{\square})$. The $K_{\mathrm{d}}$ value presented in Figure $13 \mathrm{~B}$ at $20 \times \mathrm{SO}$ is also included (gray square). (C) Apparent $K_{\mathrm{d}}$ of SN for pdTp as determined from melts at $0 \mathrm{mM}$, $380 \mu \mathrm{M}, 1.2 \mathrm{mM}$, and $3.8 \mathrm{mM}$ pdTp $(\bullet)$. The $K_{\mathrm{d}}$ value from Figure 13D at $20 \times \mathrm{SO}$ is included (gray circle).

(Figure 12C). In some systems, SO can therefore significantly perturb equilibria on which it is reporting. Further development of a detailed model of SO-dependent phenomena [e.g., SO selfassociation, SO-dependent protein (de)stabilization, and inhibition of binding by SO] may be able to quantitatively account for these observed effects and would be greatly facilitated if the molar dye concentration were known. Absent such a model, we have chosen to report $K_{\mathrm{d}}$ values at $20 \times \mathrm{SO}$, because high signalto-noise ratios are reliably observed. We acknowledge that inaccuracy may be introduced by any model that assumes that $\mathrm{SO}$ is an inert reporter of protein (un)folding and binding that does not perturb the system. Nevertheless, in our two model systems, the $K_{\mathrm{d}}$ values determined by SO-monitored thermal shift span a relatively small range over 1 order of magnitude range of SO concentrations, suggesting that neglecting these effects is a reasonable first approximation.

Experimental Case Studies: Maltose Binding Protein and Staphylococcal Nuclease. At a given SO concentration, a $K_{\mathrm{d}}$ value for maltose binding is obtained as described above with eqs $9,11,24,26$, and 31 (Figures 4 and 13A,B). This analysis at the $T_{\mathrm{m}}$ yields a $K_{\mathrm{d}}$ value of $2.9 \mu \mathrm{M}$ at $20 \times \mathrm{SO}$, varying between 0.6 and $5.0 \mu \mathrm{M}$ for experiments performed at various $\mathrm{SO}$ concentrations (Figure 12B). These values are in reasonable agreement with dissociation constants determined by equilibrium dialysis (37) at room temperature $(2.2 \mu \mathrm{M})$, or quantitative cysteine reactivity $(8)$ of single-cysteine mutants of MBP at the 

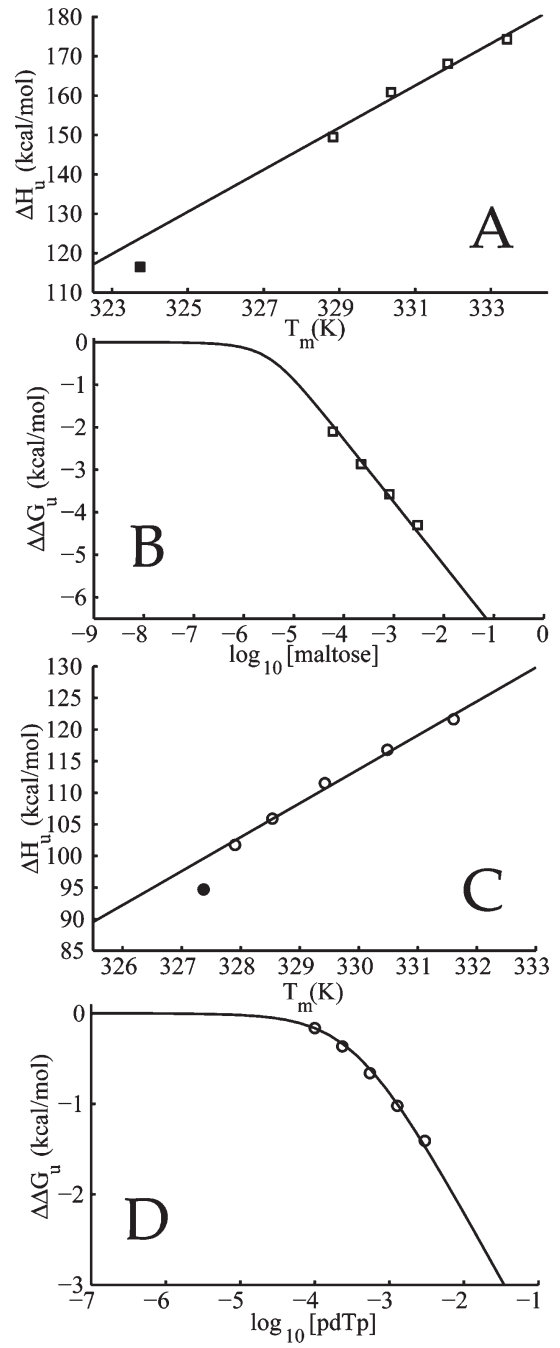

FiguRE 13: Experimental determination of $\Delta^{\mathrm{L}} C_{p, \mathrm{u}}$ and $K_{\mathrm{d}}$ values (A) Estimation of $\Delta^{\mathrm{L}} C_{p, \mathrm{u}}$ for an MBP-maltose complex (5.3 kcal $\mathrm{mol}^{-1} \mathrm{~K}^{-1}$ ) by a linear fit of $\Delta^{\mathrm{L}} H_{\mathrm{u}}$ and $T_{\mathrm{m}}$ values determined experimentally at $60,221,814$, and $3 \mu \mathrm{M}$ maltose ( $\square$ ). The value for the apoprotein (ם) falls slightly below the fit line, presumably reflecting the absence of the $\Delta H_{\mathrm{b}}$ contribution to $\Delta H_{\mathrm{u}}$ (cf. Figure 5). (B) Dependence of $\Delta \Delta G_{\mathrm{u}}$ on maltose concentration (cf. Figure 4) that can be fit (eq 25) for a $K_{\mathrm{d}}$ value of $2.9 \mu \mathrm{M}$ using the model in which maltose binds selectively to the native state for the maltose concentrations given above (cf. Figure 3B). (C and D) Analysis of pdTp binding to SN (at $100 \mu \mathrm{M}, 234 \mu \mathrm{M}, 548 \mu \mathrm{M}, 1.28 \mathrm{mM}$, and $3 \mathrm{mM}$ $\left.\operatorname{pdTp} ; \Delta^{\mathrm{L}} C_{p, \mathrm{u}}=5.4 \mathrm{kcal} \mathrm{mol}^{-1} \mathrm{~K}^{-1} ; K_{\mathrm{d}}=350 \mu \mathrm{M}\right)$. Note that the $\Delta H_{\mathrm{u}}$ of the apoprotein falls below the fit line, as for MBP, again reflecting the contribution of $\Delta H_{\mathrm{b}}$. In both cases, this deviation from the fit line is consistent with average values of $\Delta H_{\mathrm{b}}$ observed in other protein-ligand complexes of $\sim 5-15 \mathrm{kcal} / \mathrm{mol}$ (46). All experiments were conducted with $20 \times$ SO.

$T_{\mathrm{m}}(\sim 10 \mu \mathrm{M})$. For MBP, the experimentally determined value of $\Delta^{\mathrm{L}} C_{p, \mathrm{u}}$ is $5.3 \mathrm{kcal} \mathrm{mol}^{-1} \mathrm{~K}^{-1}$ at $20 \times \mathrm{SO}$ (Figure $13 \mathrm{~A}$ ), varying between 3.5 and $9.0 \mathrm{kcal} \mathrm{mol}^{-1} \mathrm{~K}^{-1}$ across the same range of SO concentrations [note that the heat capacity at $20 \times$ SO was estimated from four observations, whereas the others use only two (see Figures 12 and 13)]. For $\Delta C_{p, \mathrm{u}}$ values above $\sim 4.3 \mathrm{kcal}$ $\mathrm{mol}^{-1} \mathrm{~K}^{-1}$, eq 5 indicates that MBP should cold denature at temperatures accessible in aqueous solutions. Other studies that have experimentally determined heat capacities for $\operatorname{MBP}(33,38)$ make similar observations about the calculated "cold" $T_{\mathrm{m}}$ of MBP but find no evidence of unfolding at low temperatures, though they do find reduced stability at low temperatures as measured by chemical denaturation. The discrepancy between heat capacity and cold denaturation of MBP remains unresolved. A model that incorporates the temperature dependence of $\Delta C_{p, \mathrm{u}}$ (39) may be necessary to extrapolate from observations made at the "hot" $T_{\mathrm{m}}$ to estimate accurate temperatures of cold denaturation in this system. Even so, a temperature-independent heat capacity is probably a reasonable approximation for this application where $\Delta G_{\mathrm{u}}$ is extrapolated over a few degrees in a typical ligand-induced thermal shift.

At $20 \times \mathrm{SO}$, the $K_{\mathrm{d}}$ value of $\mathrm{SN}$ for pdTp in the absence of $\mathrm{Ca}^{2+}$ is $350 \mu \mathrm{M}$, varying between 350 and $430 \mu \mathrm{M}$ with $\mathrm{SO}$, and the $\Delta C_{p, \mathrm{u}}$ value is $5.4 \mathrm{kcal} \mathrm{mol}^{-1} \mathrm{~K}^{-1}$ (Figure 13C), varying between 4.6 and $6.9 \mathrm{kcal} \mathrm{mol}^{-1} \mathrm{~K}^{-1}$ over the SO concentration range. SN stability and affinity for pdTp were relatively insensitive to SO concentration (Figure 12A,C). Other methods reported in the literature have yielded dissociation constants of $95 \mu \mathrm{M}(40)$ and $23 \mu \mathrm{M}(8)$.

\section{DISCUSSION}

Quantification of protein-ligand interactions by the measurement of ligand-induced changes in protein stability offers a number of advantages over methods that rely on the experimental determination of the fraction of the protein-ligand complex. These advantages include a great degree of generality, a choice of techniques for measuring protein stability, and, most importantly, a very large range of protein or ligand concentrations and affinities over which the observations are informative. Provided there is no affinity of ligand for the denatured state, no limiting behavior of the thermal shift will be observed and the only theoretical limit to the affinities that can be measured by stability changes is set by the upper limit of experimentally accessible temperatures. This property is particularly invaluable for assessing very high-affinity interactions (1), which otherwise require experimentally demanding approaches (41). Low-affinity interactions are also experimentally accessible provided ligand can be added at sufficiently high concentrations.

A disadvantage of measuring affinity by ligand-induced stability changes is that a relatively sophisticated treatment of the linkage between binding and stability is necessary to interpret the data and extract the relevant parameters. We have therefore presented a thermodynamic model in some detail in an attempt to clarify the salient features. As mentioned above, the most important and unique feature of the method is that information about binding affinity can still be obtained at ligand concentrations at which the protein-ligand complex is saturated because the free energy of binding continues to increase with ligand concentration. Because the enthalpy due to binding is proportional to the fraction of protein bound by ligand (42), all such increases in $\Delta G_{\mathrm{b}}$ (and therefore $T_{\mathrm{m}}$ ) beyond the ligand concentration at which the protein-ligand complex is saturated are the result of entropic contributions.

Monitoring protein unfolding with dyes such as Sypro Orange (SO), which increase their fluorescence emission intensities upon interaction with an unfolded state, provides an experimentally straightforward, economical, and high-throughput method for observing thermal melts using commonly available RT-PCR instrumentation. However, analysis of these experimental observations requires careful consideration of the dye-mediated reporting mechanism and the underlying thermodynamic model to extract free energies of stability and ligand binding.

We have shown that SO exhibits unanticipated properties that should be taken into account in experimental design and data 
analysis. First, in the absence of protein, SO apparently selfassociates in aqueous solution. This means that changes in fluorescence intensity could have two possible origins: a passive effect in which a decrease in the free SO concentration shifts the self-association equilibrium toward the more fluorescent free monomer or an active effect in which FEI increases through direct interaction with the denatured state. Second, SO can significantly perturb the unfolding and binding equilibria on which it is reporting. Third, SO exhibits a large nonlinear intrinsic temperature dependence of fluorescence intensity, which is included in the equations used to fit experimental data.

The measurement of ligand-binding affinities exploits the linked equilibria of binding and folding manifested as a liganddependent shift in $T_{\mathrm{m}}$ values. To report ligand binding free energy at a common temperature, we calculate the ligand-mediated change in the free energy of stability at that reference temperature with the Gibbs-Helmholtz relationship using ligand-shifted $T_{\mathrm{m}}$ and $\Delta H_{\mathrm{u}}$ values. A critical parameter in the use of this equation is the change in heat capacity that occurs upon (un)folding $\left(\Delta C_{p, \mathrm{u}}\right)$. This parameter is underdetermined from fits to a single-experimental unfolding transition (4), although this approach is sometimes used $(2,3)$. It is possible to use heat capacity values calculated from the size of the protein as reasonable estimates (34). However, we take advantage of the fact that $\Delta C_{p, \mathrm{u}}$ can be obtained experimentally directly from a ligand titration series, within which each thermal melt has a unique $T_{\mathrm{m}}$ and $\Delta H_{\mathrm{u}}$, thereby providing the temperature dependence of $\Delta H_{\mathrm{u}}$ (which is the heat capacity by definition). Once $\Delta \Delta G_{\mathrm{u}}$ values have been calculated at a common temperature for various ligand concentrations, the affinity of the interaction is determined by fitting the canonical equation for the free energy of ligand binding (27). To extract $T_{\mathrm{m}}$ and $\Delta H_{\mathrm{u}}$ values from experimental observations, we have developed general methods that allow the incorporation of any baseline model (e.g., linear baseline model for CD or nonlinear for SO) directly into the equation describing the inverse first derivative of the temperature dependence of the experimental fluorescence signal.

This thermodynamic analysis implies that experimental observations are made under equilibrium conditions. We have shown that SO-monitored unfolding can be reversible, but it is also necessary to ensure that the experimental rate of temperature change is commensurate with relaxation processes that establish equilibrium (kinetics of protein folding, ligand binding, SO interaction, and thermal equilibration). We find that equilibration conditions vary dramatically from protein to protein and are dependent on ligand. It is therefore necessary to design experiments that test whether equilibration conditions are achieved with the experimental temperature ramp rate. It should be noted that $T_{\mathrm{m}}$ shifts observed at ramp rates that are too fast to allow equilibration are still useful for screening ligand binding and provide semiquantitative rank ordering of affinities. We also note that it is possible to design experiments that rely on isothermal relaxation kinetics to extract stabilities and binding constants (12). At temperatures near the $T_{\mathrm{m}}$, protein-dependent irreversible processes are at work and may preclude accurate analysis with equilibrium models for some proteins (43-45). There is a trade-off between slower ramp times allowing more equilibration time and faster ramp rates minimizing aggregation and misfolding.

The application of this method to thermophilic proteins may be infeasible if the $T_{\mathrm{m}}$ values exceed the experimentally addressable range. In principle, it is possible to lower the $T_{\mathrm{m}}$ through the use of chemical denaturants such as urea; however, we find that these reagents may also alter the fluorescence emission intensity of SO (Figure 6). Destabilizing mutagenesis may be used to bring thermophilic proteins into an experimentally accessible regime.

Given its high environmental sensitivity, SO fluorescence in the presence of both native and denatured protein varies greatly with different proteins. To monitor protein unfolding, the total SO concentration and protein concentration $\left([\mathrm{P}]_{\mathrm{T}}\right)$ should be empirically minimized to the extent possible without loss of signal, both to reduce irreversibility through aggregation effects and to maximize the information obtained from ligand binding experiments. Although the binding model can account for stoichiometric binding (eq 23), if $[\mathrm{L}]_{\mathrm{T}} \ll[\mathrm{P}]_{\mathrm{T}}, T_{\mathrm{m}}$ shifts will be negligible; if $[\mathrm{L}]_{\mathrm{T}} \approx[\mathrm{P}]_{\mathrm{T}}, T_{\mathrm{m}}$ shifts contain little reliable information about $K_{\mathrm{d}}$ (cf. Figure $3 \mathrm{C}$ ). Affinities are most reliably determined if $[\mathrm{L}]_{\mathrm{T}} \gg[\mathrm{P}]_{\mathrm{T}}$ (by $\sim 1$ order of magnitude). In this study, protein concentrations between 2 and $6 \mu \mathrm{M}$ were used. We anticipate that monitoring will be possible with lower protein concentrations as more sensitive detection methods are developed, further diminishing scale and minimizing irreversible offpathway processes.

It is remarkably straightforward to collect experimental thermal melt data using extrinsic dyes that fluoresce upon interaction with unfolded protein. Great care must be exercised in experimental design and quantitative analysis of the data (e.g., to account for effects the dye may have on the equilibria under observation as we illustrate for Sypro Orange). Nevertheless, these methods enable experimental observation of many fundamental thermodynamic parameters (e.g., $K_{\mathrm{d}}, \Delta G_{\mathrm{u}}, \Delta G_{\mathrm{b}}, \Delta H_{\mathrm{u}}$, $\Delta H_{\mathrm{b}}$, and $\Delta^{\mathrm{L}} C_{p, \mathrm{u}}$ ) with unprecedented ease and throughput. Their continued development is likely to play an important role in the analysis of biological systems.

\section{ACKNOWLEDGMENT}

We acknowledge Terry Oas for many helpful discussions and Dan Isom and Phillipe Marguet for discussions and the gift of purified SN and RBP.

\section{SUPPORTING INFORMATION AVAILABLE}

Gene sequences of SN, MBP, and RBP, the derivation of eq 23 , and a more detailed description of the procedure used for fitting eq 31 to experimental data generated with SO. This material is available free of charge via the Internet at http://pubs.acs.org.

\section{REFERENCES}

1. Brandts, J. F., and Lin, L. N. (1990) Study of strong to ultratight protein interactions using differential scanning calorimetry. Biochemistry 29, 6927-6940.

2. Pantoliano, M. W., Petrella, E. C., Kwasnoski, J. D., Lobanov, V. S., Myslik, J., Graf, E., Carver, T., Asel, E., Springer, B. A., Lane, P., and Salemme, F. R. (2001) High-density miniaturized thermal shift assays as a general strategy for drug discovery. J. Biomol. Screening 6 429-440.

3. Lo, M., Aulabaugh, A., Jin, G., Cowling, R., Bard, J., Malamas, M., and Ellestad, G. (2004) Evaluation of fluorescence-based thermal shift assays for hit identification in drug discovery. Anal. Biochem. 332, $153-159$.

4. Matulis, D., Kranz, J. K., Salemme, F. R., and Todd, M. J. (2005) Thermodynamic stability of carbonic anhydrase: Measurements of binding affinity and stoichiometry using ThermoFluor. Biochemistry 44, 5258-5266.

5. Cimmperman, P., Baranauskiene, L., Jachimoviciūte, S., Jachno, J., Torresan, J., Michailoviene, V., Matuliene, J., Sereikaite, J., Bumelis, V., and Matulis, D. (2008) A quantitative model of thermal stabilization and destabilization of proteins by ligands. Biophys. J. 95, 3222-3231. 
6. Waldron, T. T., and Murphy, K. P. (2003) Stabilization of proteins by ligand binding: Application to drug screening and determination of unfolding energetics. Biochemistry 42, 5058-5064.

7. Pace, C. N., and McGrath, T. (1980) Substrate stabilization of lysozyme to thermal and guanidine hydrochloride denaturation. J. Biol. Chem. 255, 3862-3865.

8. Isom, D. G., Vardy, E., Oas, T. G., and Hellinga, H. W. (2010) Picomole-Scale Characterization of Protein Stability and Function by Quantitative Cysteine Reactivity. Proc. Natl. Acad. Sci. U.S.A. 107, 4908-4913.

9. Ghaemmaghami, S., Fitzgerald, M. C., and Oas, T. G. (2000) A quantitative, high-throughput screen for protein stability. Proc. Natl. Acad. Sci. U.S.A. 97, 8296-8301.

10. Park, C., and Marqusee, S. (2005) Pulse proteolysis: A simple method for quantitative determination of protein stability and ligand binding. Nat. Methods 2, 207-212.

11. West, G. M., Tang, L., and Fitzgerald, M. C. (2008) Thermodynamic analysis of protein stability and ligand binding using a chemical modification- and mass spectrometry-based strategy. Anal. Chem. 80, 4175-4185.

12. Senisterra, G. A., and Finerty, P. J. (2009) High throughput methods of assessing protein stability and aggregation. Mol. Biosyst. 5, 217223.

13. Niesen, F. H., Berglund, H., and Vedadi, M. (2007) The use of differential scanning fluorimetry to detect ligand interactions that promote protein stability. Nat. Protoc. 2, 2212-2221.

14. Steinberg, T. H., Jones, L. J., Haugland, R. P., and Singer, V. L. (1996) SYPRO orange and SYPRO red protein gel stains: One-step fluorescent staining of denaturing gels for detection of nanogram levels of protein. Anal. Biochem. 239, 223-237.

15. Cummings, M., Farnum, M., and Nelen, M. (2006) Universal screening methods and applications of ThermoFluor. J. Biomol. Screening $11,854-863$.

16. Ericsson, U. B., Hallberg, B. M., Detitta, G. T., Dekker, N., and Nordlund, P. (2006) Thermofluor-based high-throughput stability optimization of proteins for structural studies. Anal. Biochem. 357, 289-298.

17. Vedadi, M., Niesen, F. H., Allali-Hassani, A., Fedorov, O. Y., Finerty, P. J., Wasney, G. A., Yeung, R., Arrowsmith, C., Ball, L. J., Berglund, H., Hui, R., Marsden, B. D., Nordlund, P., Sundstrom, M., Weigelt, J., and Edwards, A. M. (2006) Chemical screening methods to identify ligands that promote protein stability, protein crystallization, and structure determination. Proc. Natl. Acad. Sci. U.S.A. $103,15835-15840$.

18. Lavinder, J. J., Hari, S. B., Sullivan, B. J., and Magliery, T. J. (2009) High-throughput thermal scanning: A general, rapid dye-binding thermal shift screen for protein engineering. J. Am. Chem. Soc. 131, 3794-3795.

19. Gasteiger, E., Hoogland, C., Gattiker, A., Duvaud, S., Wilkins, M. R., Appel, R. D., and Bairoch, A. (2005) Single-Protein Analysis Tools on the ExPASy Server. In The Proteomics Protocols Handbook, pp 575-582, Humana Press, Totowa, NJ.

20. Parker, D., Davis, A., and Taniuchi, H. (1981) Further study of the conformation of nuclease-(1-126) in relation to intrinsic enzymatic activity. J. Biol. Chem. 256, 4557-4569.

21. Privalov, P. L., and Khechinashvili, N. N. (1974) A thermodynamic approach to the problem of stabilization of globular protein structure: A calorimetric study. J. Mol. Biol. 86, 665-684.

22. Privalov, P. L., and Gill, S. J. (1988) Stability of protein structure and hydrophobic interaction. Adv. Protein Chem. 39, 191-234.

23. Privalov, P. L. (1990) Cold Denaturation of Protein. Crit. Rev. Biochem. Mol. Biol. 25, 281-306.

24. Marky, L. A., and Breslauer, K. J. (1987) Calculating thermodynamic data for transitions of any molecularity from equilibrium melting curves. Biopolymers 26, 1601-1620.
25. John, D. M., and Weeks, K. M. (2000) van't Hoff enthalpies without baselines. Protein Sci. 9, 1416-1419.

26. Allen, D. L., and Pielak, G. J. (1998) Baseline length and automated fitting of denaturation data. Protein Sci. 7, 1262-1263.

27. Schellman, J. A. (1975) Macromolecular binding. Biopolymers 14, 999-1018.

28. Wyman, J., and Gill, S. J. (1990) Binding and linkage: Functional chemistry of biological macromolecules, University Science Books, Mill Valley, CA.

29. Wyman, J. (1974) A Probabilistic Approach to Cooperativity of Ligand Binding by a Polyvalent Molecule. Proc. Natl. Acad. Sci. U.S.A. 71, 3431-3434.

30. Cimmperman, P., Baranauskiene, L., Jachimoviciūte, S., Jachno, J., Torresan, J., Michailoviene, V., Matuliene, J., Sereikaite, J., Bumelis, V., and Matulis, D. (2008) A quantitative model of thermal stabilization and destabilization of proteins by ligands. Biophys. J. 95, 32223231.

31. Pace, C. N., Grimsley, G. R., Thomas, S. T., and Makhatadze, G. I. (1999) Heat capacity change for ribonuclease A folding. Protein Sci.8, $1500-1504$.

32. Nakamura, S., and Kidokoro, S. (2004) Isothermal acid-titration calorimetry for evaluating the $\mathrm{pH}$ dependence of protein stability. Biophys. Chem. 109, 229-249.

33. Ganesh, C., Shah, A. N., Swaminathan, C. P., Surolia, A., and Varadarajan, R. (1997) Thermodynamic Characterization of the Reversible, Two-State Unfolding of Maltose Binding Protein, a Large Two-Domain Protein. Biochemistry 36, 5020-5028.

34. Myers, J. K., Pace, C. N., and Scholtz, J. M. (1995) Denaturant m values and heat capacity changes: Relation to changes in accessible surface areas of protein unfolding. Protein Sci. 4, 2138-2148.

35. Hawe, A., Sutter, M., and Jiskoot, W. (2008) Extrinsic fluorescent dyes as tools for protein characterization. Pharm. Res. 25, $1487-1499$

36. Martin, R. B. (1996) Comparisons of Indefinite Self-Association Models. Chem. Rev. 96, 3043-3064.

37. Schwartz, M., Kellermann, O., Szmelcman, S., and Hazelbauer, G. L. (1976) Further Studies on the Binding of Maltose to the MaltoseBinding Protein of Escherichia coli. Eur. J. Biochem. 71, 167-170.

38. Sheshadri, S., Lingaraju, G. M., and Varadarajan, R. (1999) Denaturant mediated unfolding of both native and molten globule states of maltose binding protein are accompanied by large $\Delta \mathrm{Cp}$ 's. Protein $S c i$. $8,1689-1695$

39. Becktel, W. J., and Schellman, J. A. (1987) Protein stability curves. Biopolymers 26, 1859-1877.

40. Serpersu, E. H., Shortle, D., and Mildvan, A. S. (1986) Kinetic and magnetic resonance studies of effects of genetic substitution of a calcium-liganding amino acid in staphylococcal nuclease. Biochemistry 25, 68-77.

41. Boder, E. T., Midelfort, K. S., and Wittrup, K. D. (2000) Directed evolution of antibody fragments with monovalent femtomolar antigen-binding affinity. Proc. Natl. Acad. Sci. U.S.A. 97, 1070110705.

42. Leavitt, S., and Freire, E. (2001) Direct measurement of protein binding energetics by isothermal titration calorimetry. Curr. Opin. Struct. Biol. 11, 560-566.

43. Apenten, R. (1998) The effect of protein unfolding stability on their rates of irreversible denaturation. Food Hydrocolloids 12, 1-8.

44. Sturtevant, J. (1987) Biochemical applications of differential scanning calorimetry. Annu. Rev. Phys. Chem. 38, 463-488.

45. Privalov, P. (1982) Stability of Proteins: Proteins which do not Present a Single Cooperative System. Adv. Protein Chem. 35, 28-29.

46. Biltonen, R., and Langerman, N. (1979) Microcalorimetry for biological chemistry: Experimental design, data analysis, and interpretation. Methods Enzymol. 61, 287-318. 